\title{
Effects of frontal transcranial direct current stimulation on emotional state and processing in healthy humans
}

\author{
M. A. Nitsche ${ }^{1}$, J. Koschack ${ }^{2}$, H. Pohlers' ${ }^{1}$, S. Hullemann ${ }^{1}$, W. Paulus ${ }^{1}$ and S. Happe ${ }^{1}$ \\ 1 Department Clinical Neurophysiology, Georg-August-University, Goettingen, Germany \\ ${ }^{2}$ Department General Practice/Family Medicine, Georg-August-University, Goettingen, Germany
}

\section{Edited by:}

Paulo Sérgio Boggio, Mackenzie

Presbyterian University, Brazil

Reviewed by:

Carlo Miniussi, University of Brescia, Italy

Sandra Carvalho, Universidade do Minho, Portugal

${ }^{*}$ Correspondence:

M. A. Nitsche, Department Clinical Neurophysiology,

Georg-August-University,

Robert-Koch-Str. 40, 37099

Goettingen, Germany.

e-mail:mnitsch1@gwdg.de
The prefrontal cortex is involved in mood and emotional processing. In patients suffering from depression, the left dorsolateral prefrontal cortex (DLPFC) is hypoactive, while activity of the right DLPFC is enhanced. Counterbalancing these pathological excitability alterations by repetitive transcranial magnetic stimulation (rTMS) or transcranial direct current stimulation (tDCS) improves mood in these patients. In healthy subjects, however, rTMS of the same areas has no major effect, and the effects of tDCS are mixed. We aimed to evaluate the effects of prefrontal tDCS on emotion and emotion-related cognitive processing in healthy humans. In a first study, we administered excitability-enhancing anodal, excitability-diminishing cathodal, and placebo tDCS to the left DLPFC, combined with antagonistic stimulation of the right frontopolar cortex, and tested acute emotional changes by an adjective checklist. Subjective emotions were not influenced by tDCS. Emotional face identification, however, which was explored in a second experiment, was subtly improved by a tDCS-driven excitability modulation of the prefrontal cortex, markedly by anodal tDCS of the left DLPFC for positive emotional content. We conclude that tDCS of the prefrontal cortex improves emotion processing in healthy subjects, but does not influence subjective emotional state.

Keywords: emotion, prefrontal cortex, face recognition, brain, human

\section{INTRODUCTION}

The prefrontal cortex takes part in the neuronal networks involved in mood and emotion processing. Hereby emotion can be defined as a relatively brief, reactive, and intensive emotional state, whereas mood is a more stable, constant, and less reactive emotional state (Ekman, 1999; Ellis and Moore, 1999). In healthy subjects, the ventromedial, and inferior-medial prefrontal cortex are prominently involved in self-referenced affective state (Phan et al., 2002; Steele and Lawrie, 2004). The dorsolateral prefrontal cortex (DLPFC) is more involved in processing of stimuli with not self-referential emotional content, e.g., faces or visual scenes (Ueda et al., 2003; Sergerie et al., 2005; Grimm et al., 2006). However, this distinction, which reflects the fact that the medial prefrontal cortex is generally more heavily involved in emotional, and the lateral prefrontal cortex in cognitive processing, is gradual (Steele and Lawrie, 2004). Moreover, a hemispherical difference of processing of positive and negative emotional content has been described. Happy mood and positive emotional stimuli induce predominant left DPLFC activity (Habel et al., 2005; Herrington et al., 2005; Sergerie et al., 2005). In accordance, lesions of the left prefrontal cortex by stroke, tumors, or epilepsy are often accompanied by depression, while lesions of the right prefrontal cortex are associated with elated mood (Robinson and Lipsey, 1985; Perini, 1986; Belyi, 1987). Clinical depression is associated with left DLPFC hypoactivity, while activity of the right prefrontal cortex might be increased (Schutter and van Honk, 2005).
Consequently, it has been proposed that an activation of the left DLPFC might turn mood and emotion into more positive states. Indeed, activity-enhancing repetitive transcranial magnetic stimulation (rTMS) improves symptoms of depressed patients (Mitchell and Loo, 2006). A similar result was found for excitabilityenhancing transcranial direct current stimulation (tDCS; Fregni et al., 2006). Moreover, excitability-enhancing tDCS improved performance in an affective go-non-nongo task for positive emotional content in depressed subjects (Boggio et al., 2007). tDCS induces long-lasting, stimulation polarity-dependent excitability shifts of the human cerebral cortex via neuronal de- or hyperpolarization and the subsequent modification of NMDA receptor strength (Nitsche and Paulus, 2000, 2001; Nitsche et al., 2003a,b).

Enhancing excitability of the left DLPFC in healthy humans by rTMS so far failed to induce a positive shift of emotional state (Mosimann et al., 2000; Baeken et al., 2006). However left prefrontal rTMS was able to modulate mood-related information processing (Schutter and van Honk, 2006). Studies exploring alterations of emotional state by prefrontal tDCS in healthy subjects show mixed results. Plazier et al. (in press) describe no effects of prefrontal tDCS on subjective mood. However, the emotional valence of unpleasant pictures was diminished via left DLPFC anodal tDCS (Boggio et al., 2009; Maeoka et al., 2012).

In the present study, we aimed to disentangle the effect of prefrontal tDCS on subjective emotional state and emotional staterelated information processing in healthy humans. In the first experiment, we tested the effect of excitability-enhancing anodal 
tDCS, excitability-diminishing cathodal tDCS, or sham tDCS of the left DLPFC (combined with antagonistic stimulation of the right frontopolar cortex) on self-referenced emotional state via a visual analog scale (VAS). If tDCS works similarly in healthy subjects and depressed patients, anodal tDCS should shift emotional state to more positive values. In the second experiment, we tested the effect of the identical tDCS protocols on not self-referenced emotional state-related information processing. DLPFC function might be involved more in the latter kind of tasks than in actual modulation of emotional state in healthy subjects. Moreover, the left DLPFC is important for the processing of positive affects. Thus we hypothesized a positive effect of excitability-enhancing tDCS for emotionally positive material. Since the main aim of this study was to explore the effects of a tDCS protocol used for the treatment of depression on emotional state and emotional processing in healthy individuals, we performed only left DLPFC anodal stimulation, and did not explore the effects of right DLPFC anodal tDCS, which might result in antagonistic effects.

\section{MATERIALS AND METHODS \\ SUBJECTS}

Fourteen healthy volunteers (five female, mean age $33.29 \pm 8.49$ SD) participated in Experiment 1. Seventeen subjects participated in Experiment 2 (eight female, mean age 24.88 \pm 2.34 SD). All gave written informed consent. The investigation was approved by the ethics committee of the University of Goettingen, and the experiments conform to the principles laid down in the Declaration of Helsinki.

\section{tDCS OF THE DLPFC}

Current $(1 \mathrm{~mA})$ was induced through saline-soaked sponge electrodes (surface $35 \mathrm{~cm}^{2}$ ), resulting in a current density of $0.0286 \mathrm{~mA} / \mathrm{cm}^{2}$. tDCS was delivered by a specially developed, battery-driven constant-current stimulator (Schneider Electronic, Gleichen, Germany). Current strength was ramped up in the first $10 \mathrm{~s}$ of tDCS and turned off the same way to avoid phosphene perception and diminish tingling sensations. For placebo tDCS, current flow was terminated after $20 \mathrm{~s}$. These stimulation characteristics reliably allow placebo stimulation, i.e., subjects are not able to discriminate real from sham stimulation (Gandiga et al., 2006). In Experiment 1, real tDCS was delivered for $20 \mathrm{~min}$, in Experiment 2 for $10 \mathrm{~min}$. Former experiments have shown that these stimulation durations induce cortical excitability shifts stable for at least $1 \mathrm{~h}$ after the end of tDCS (Nitsche and Paulus, 2001; Nitsche et al., 2003a). We applied 20 min stimulation in Experiment 1 , because this is the usual stimulation duration performed for the treatment of depression (Fregni et al., 2006). tDCS duration in Experiment 2 was 10 min, because this was the duration of the face recognition task, during which tDCS was performed. The left DLPFC electrode (to which the terms anodal and cathodal stimulation refer to) was placed at F3 (international 10-20 system) and the return electrode above the contralateral orbit in both experiments.

\section{OUESTIONNAIRES}

For evaluation of emotional state, a questionnaire (Skala zur Einschätzung der Stimmung, SES; Hampel, 1977) was used. The
SES is a VAS in German language, which contains adjectives representing happy and sad emotional state as well as lethargy (neutral mood condition). Fourteen adjectives per category were included. The VAS scale ranges from 1 (absent) to 7 (maximum strength). In difference to the more widely used PANAS (Watson and Clark, 1988), which was developed to obtain dispositional affect measures over the last 12 months for positive and negative mood, the SES specifically measures actually present emotion, and adds another emotional dimension, i.e., the neutral emotion condition. Moreover, the VAS of the SES contains seven stages, that of the PNAS only five. We chose the SES instead of the more widely used PANAS, because it is explicitly validated for present emotional state. A limitation of this choice might be the limited comparability with other studies, in which the PANAS was applied. For assessment of depression, subjects performed the Beck Depression Inventory (BDI), and the Hamilton Depression rating scale (HAMD). The BDI is a 21-item self-assessment test presented in multiplechoice format, which measures the presence and the degree of depression in adults (Beck et al., 1961). The HAMD is a 21item peer-evaluation test, which rates the presence of depressive symptoms as established in a clinical interview (Hamilton, 1960).

\section{EMOTIONAL FACE RECOGNITION TASK}

In this task, two emotional expressions of a human face are simultaneously presented on a computer monitor, one joy, or anger, and the other neutral.

Subjects were instructed to identify as fast as possible the position of the emotionally positive or negative facial expression and to press the appropriate button on a keyboard.

All stimuli used in the study were part of Ekman's series of pictures of facial affect (Ekman and Friesen, 1975, 1976). They were presented by a DOS-based software for creating, conducting, and analyzing reaction time tasks (Experimental Run Time System ${ }^{\odot}$, BeriSoft Cooperation) on a IBM-compatible computer connected with a $21^{\prime \prime}$ monitor.

Every trial consisted of the simultaneous presentation of two pictures on the left and right side of the screen for $50 \mathrm{~ms}$. The two pictures showed the same person, on one side with an emotional expression (positive, i.e., joy, or negative, i.e., anger) as the target stimulus, on the other side with a neutral facial expression. The two pictures were followed by two question marks presented in place of the facial affect stimuli. Subjects were instructed to judge on which side of the screen the face with an emotional expression had been shown by pressing the left or right button on the keyboard. A red colored cross was shown for $1000 \mathrm{~ms}$ to mark the beginning of a trial and to make the subject fixate the center of the screen. Figure 1 shows an example of the trial structure.

Twelve different trial conditions emerged from randomly varying the position of the target stimulus (left; right), the emotional value of the target stimulus (positive; negative), and the identity of the person on the pictures (female; male no. 1 ; male no. 2). Each trial condition was shown for four times within a session. Each session consisted of 50 trials, with two randomly chosen warm-up trials not being included in the statistical analysis and 48 permutated experimental trials. 


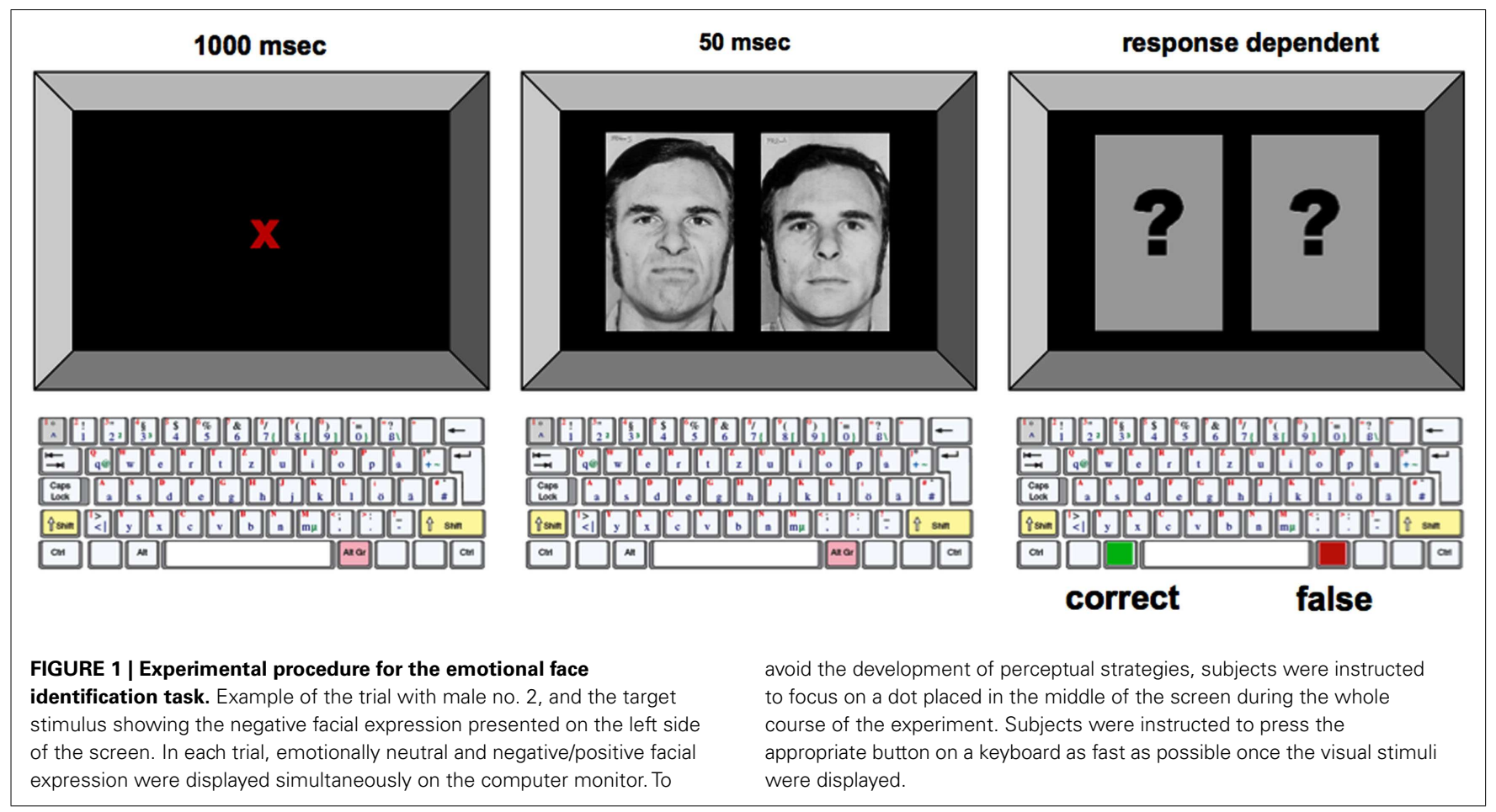

\section{EXPERIMENTAL PROCEDURES}

\section{Experiment 1}

To exclude a state of clinical depression in our healthy subjects, HAMD and BDI were performed before the start of each experimental session.

Afterward, subjects evaluated emotional state before and repetitively after anodal, cathodal, or placebo tDCS. The order of application of the tDCS session was randomized. A complete crossover design was performed. Between each tDCS session, a break of at least 1 week was obligatory to avoid interference effects of stimulation.

Subjects were seated in front of a table, and the position of F3 was identified according to the international 1020 system. Afterward, the SES was handed out. Subjects were specifically instructed to evaluate their actual emotional state. After baseline measures, the tDCS electrodes were fixed onto the head and tDCS was performed for $20 \mathrm{~min}$ with $1 \mathrm{~mA}$ current strength. Immediately after the end of tDCS subjects performed the SES again. SES-based evaluation of emotional state was repeated every $15 \mathrm{~min}$ for up to $1 \mathrm{~h}$ after the end of tDCS and each hour after tDCS for the next $5 \mathrm{~h}$. A last emotional state evaluation was performed the morning after stimulation.

\section{Experiment 2}

In this experiment, subjects had to identify the position of the "emotional" facial expression on a computer screen as fast as possible before and repetitively after anodal, cathodal, or placebo tDCS. The order of application of the tDCS sessions was randomized. A complete crossover design was performed. Between each tDCS session, a break of 1 week was obligatory to avoid interference effects of stimulation.
Subjects were seated in front of the screen (eye-screen distance about $75 \mathrm{~cm}$, visual angle approximately $60^{\circ}$ in width and height), the position of the tDCS electrodes was identified and the electrodes fixed onto the head. They were instructed that they should identify the position of the emotionally not neutral face out of two simultaneously displayed faces on the computer screen as fast as possible while fixating a dot positioned in the middle of the screen and press the appropriate button on the keyboard as fast as possible. Afterward, all faces were presented once and a short trial run was performed to ensure that the subjects had understood the task. Before tDCS, one baseline session was performed. Then tDCS was started and continued for $10 \mathrm{~min}$. During tDCS, two face recognition sessions were performed, one 2 min after the start of tDCS, the other after $6 \mathrm{~min}$. The remaining face recognition sessions were performed immediately after the end, and 5, 10, 20, 30 , and $60 \mathrm{~min}$ after the end of tDCS.

\section{CALCULATIONS AND STATISTICS Experiment 1}

For the SES, the sum of the VAS values for each emotional condition (neutral, positive, negative) was calculated intraindividually for each time point/tDCS condition combination. To rule out a systematic influence of subtle baseline differences on the results, the post-tDCS values were standardized by calculating the quotient of post-tDCS values vs baseline measures. Repeated measure ANOVAs were calculated for the absolute and standardized values (repeated measure factors tDCS, emotion, time course, dependent variable VAS score). In case of significant results in the ANOVA, post hoc Student's $t$-tests (repeated measures, two-tailed) were calculated to identify significant mood differences for each time point vs baseline for each tDCS condition, and differences between tDCS conditions (anodal/cathodal vs sham stimulation) for each 
time point. Critical level of significance was set to 0.05 for all calculations. The post hoc tests were not corrected for multiple comparisons.

\section{Experiment 2}

Individual means of reaction times were calculated for positive and negative affective face recognition for each tDCS condition and time point separately. Only correct trials were included in the calculations. To exclude a systematic influence of subtle baseline differences on the results, the post-tDCS values were standardized by calculating the quotient of during- and post-tDCS values vs baseline measures. Data were pooled for the two measures during tDCS, measures immediately and 5 min after tDCS, 10 and $20 \mathrm{~min}$ after tDCS and 30 and $60 \mathrm{~min}$ after tDCS. Repeated measure ANOVAs were calculated for the standardized values (repeated measure factors tDCS, emotion, time course, dependent variable reaction time). In case of significant results of the ANOVA, post hoc Student's $t$-tests (repeated measures, two-tailed) were added to identify significant mood differences for each time point dependent on tDCS condition, and to compare baseline performance. The critical $p$-value was set to 0.05 for all calculations. The post hoc tests were not corrected for multiple comparisons.

The same calculations were performed for the count of correct answers.

\section{RESULTS \\ EXPERIMENT 1}

For the healthy subjects, the mean BDI values were between 0.79 and 1.5 and the range of the mean HAMD values was $0.93-1.07$ for all stimulation conditions. These were identical between the respective tDCS conditions according to the results of the $t$-tests $(p>0.05)$.

\section{Skala zur Einschätzung der Stimmung}

For the healthy subjects, the ANOVA (absolute values) revealed significant effects of emotion and the interaction of $\mathrm{tDCS} \times$ time, but the interactions tDCS $\times$ emotion and time $\times$ tDCS $\times$ emotion, which would have revealed an impact of tDCS on emotional state, were not significant. For the standardized values, however, additionally the main effect for time and the interactions time $\times$ emotion, time $\times \mathrm{tDCS}$, and time $\times$ emotion $\times \mathrm{tDCS}$ were significant (Table 1). Comparing effects of anodal and cathodal tDCS with placebo stimulation for each time point after tDCS separately however did not reveal significant effects of tDCS on neutral, positive, or negative emotional state. As can be seen from Figure 2, in all stimulation conditions the healthy subjects rated neutral and negative mood items near the minimum, while positive adjectives were rated generally on a much higher level throughout the experiment.

Baseline values of each emotional quality did not differ significantly between tDCS sessions.

\section{EXPERIMENT 2}

\section{Reaction times}

The ANOVA revealed a significant main effect of time (Table 1). An additional trend for an effect of tDCS on performance was identified. As depicted in Figure 3, reaction times diminished throughout the course of the experiment in all tDCS and facial expression
Table 1 | Results of the ANOVAs conducted for the SES and emotional face identification task.

\begin{tabular}{|c|c|c|c|c|c|}
\hline Variables & d.f. & d.f.error & $F$-value & $p$ & $\eta^{2}$ \\
\hline \multicolumn{6}{|l|}{ EXPERIMENT 1} \\
\hline \multicolumn{6}{|l|}{ SES, absolute values } \\
\hline tDCS & 2 & 26 & 2.148 & 0.137 & 0.142 \\
\hline Emotion & 2 & 26 & 218.943 & $<0.001^{*}$ & 0.944 \\
\hline Time & 11 & 143 & 0.924 & 0.519 & 0.066 \\
\hline tDCS $\times$ emotion & 2 & 52 & 0.953 & 0.441 & 0.068 \\
\hline tDCS $\times$ time & 22 & 286 & 1.862 & $0.012^{*}$ & 0.125 \\
\hline Emotion $\times$ time & 22 & 286 & 1.305 & 0.166 & 0.091 \\
\hline tDCS $\times$ emotion $\times$ time & 44 & 572 & 1.277 & 0.114 & 0.089 \\
\hline \multicolumn{6}{|c|}{ SES, standardized values } \\
\hline tDCS & 2 & 26 & 2.431 & 0.108 & 0.158 \\
\hline Emotion & 2 & 26 & 23.358 & $<0.001^{*}$ & 0.642 \\
\hline Time & 11 & 143 & 80.405 & $<0.001^{*}$ & 0.861 \\
\hline tDCS $\times$ emotion & 2 & 52 & 0.732 & 0.574 & 0.053 \\
\hline tDCS $\times$ time & 22 & 286 & 2.017 & $0.005^{*}$ & 0.134 \\
\hline Emotion $\times$ time & 22 & 286 & 68.022 & $<0.001^{*}$ & 0.840 \\
\hline tDCS $\times$ emotion $\times$ time & 44 & 572 & 1.520 & $0.019 *$ & 0.105 \\
\hline \multicolumn{6}{|l|}{ EXPERIMENT 2} \\
\hline \multicolumn{6}{|c|}{ Standardized reaction times } \\
\hline tDCS & 2 & 40 & 2.964 & 0.063 & 0.129 \\
\hline Emotion & 1 & 20 & 1.527 & 0.231 & 0.071 \\
\hline Time & 4 & 80 & 18.713 & $<0.001^{*}$ & 0.483 \\
\hline tDCS $\times$ emotion & 2 & 40 & 1.780 & 0.182 & 0.082 \\
\hline tDCS $\times$ time & 8 & 160 & 1.444 & 0.182 & 0.067 \\
\hline Emotion $\times$ time & 4 & 80 & 2.205 & 0.076 & 0.099 \\
\hline tDCS $\times$ emotion $\times$ time & 8 & 160 & 1.159 & 0.327 & 0.055 \\
\hline \multicolumn{6}{|c|}{ Standardized correct answers } \\
\hline tDCS & 2 & 40 & 2.244 & 0.119 & 0.256 \\
\hline Emotion & 1 & 20 & 1.884 & 0.185 & 0.086 \\
\hline Time & 4 & 80 & 14.991 & $<0.001^{*}$ & 0.754 \\
\hline tDCS $\times$ emotion & 2 & 40 & 0.542 & 0.586 & 0.053 \\
\hline tDCS $\times$ time & 8 & 160 & 0.945 & 0.481 & 0.329 \\
\hline Emotion $\times$ time & 4 & 80 & 2.237 & 0.072 & 0.287 \\
\hline tDCS $\times$ emotion $\times$ time & 8 & 160 & 0.325 & 0.955 & 0.135 \\
\hline
\end{tabular}

For the SES, ANOVAs were calculated for absolute and standardized values. For the emotional face identification task, ANOVAs were calculated for standardized reaction times and number of correct answers. The asterisks mark significant main effects and interactions. d.f., degrees of freedom; F, F-value; p, probability; $\eta^{2}$, effect size.

conditions. We conducted exploratory, subjected to confirmation, post hoc $t$-tests despite only trend wise effects of tDCS or the interactions including tDCS in the ANOVA. These revealed significant shortenings of reaction time relative to baseline during and after anodal tDCS for positive and negative emotional expressions. For cathodal $\mathrm{tDCS}$, the direction of the improvements of reaction time were similar, but somewhat smaller as compared to anodal tDCS for positive emotional expressions. Conversely, under placebo stimulation the reaction time improvements occurred later during the course of the experiment and were significant - as compared to baseline - only for the last measures. The post hoc tests additionally revealed significant reaction time differences for anodal tDCS vs 


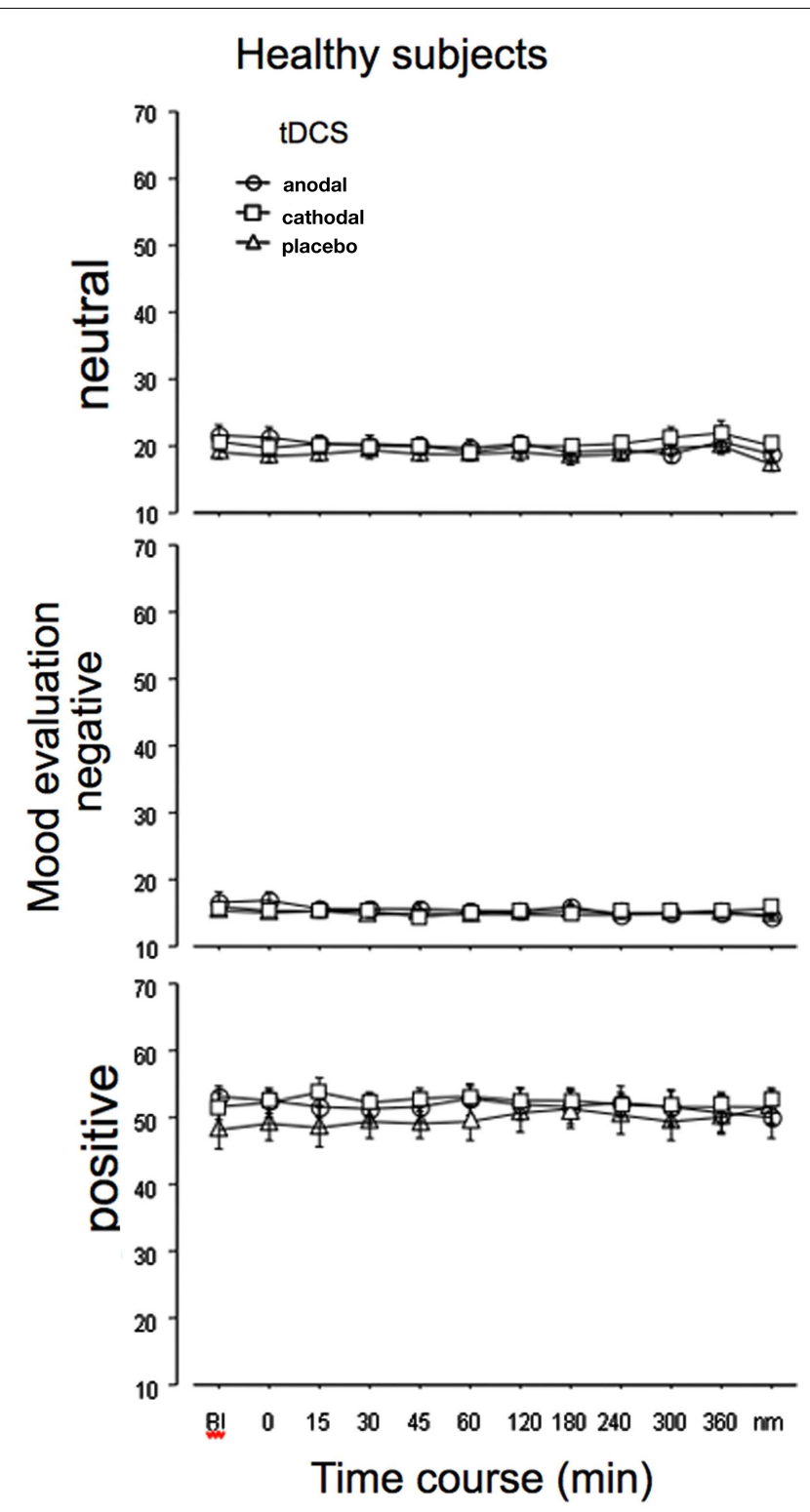

FIGURE 2 | Emotional state is not affected by tDCS in healthy subjects. Depicted are the absolute mean values before and up to the morning after anodal, cathodal, or sham tDCS over the left DLPFC for neutral (lethargic), negative, and positive emotional state, as measured by the adjective checklist SES. As can be seen from the results, the evaluation is quite stable in all stimulation conditions throughout the course of the experiment. The vertical bars indicate standard error of mean. $\mathrm{nm}$, next morning.

placebo stimulation. Anodal tDCS reduced reaction time significantly during tDCS relative to placebo stimulation for emotionally negative faces. For emotionally positive facial expressions, this effect emerged during tDCS, and remained significant for up to 10 min after tDCS. Reaction times under cathodal tDCS did differ significantly relative to placebo stimulation only for negative facial expressions during the second measures after tDCS.

Baseline performance was identical for all tDCS conditions in relation to one facial expression.

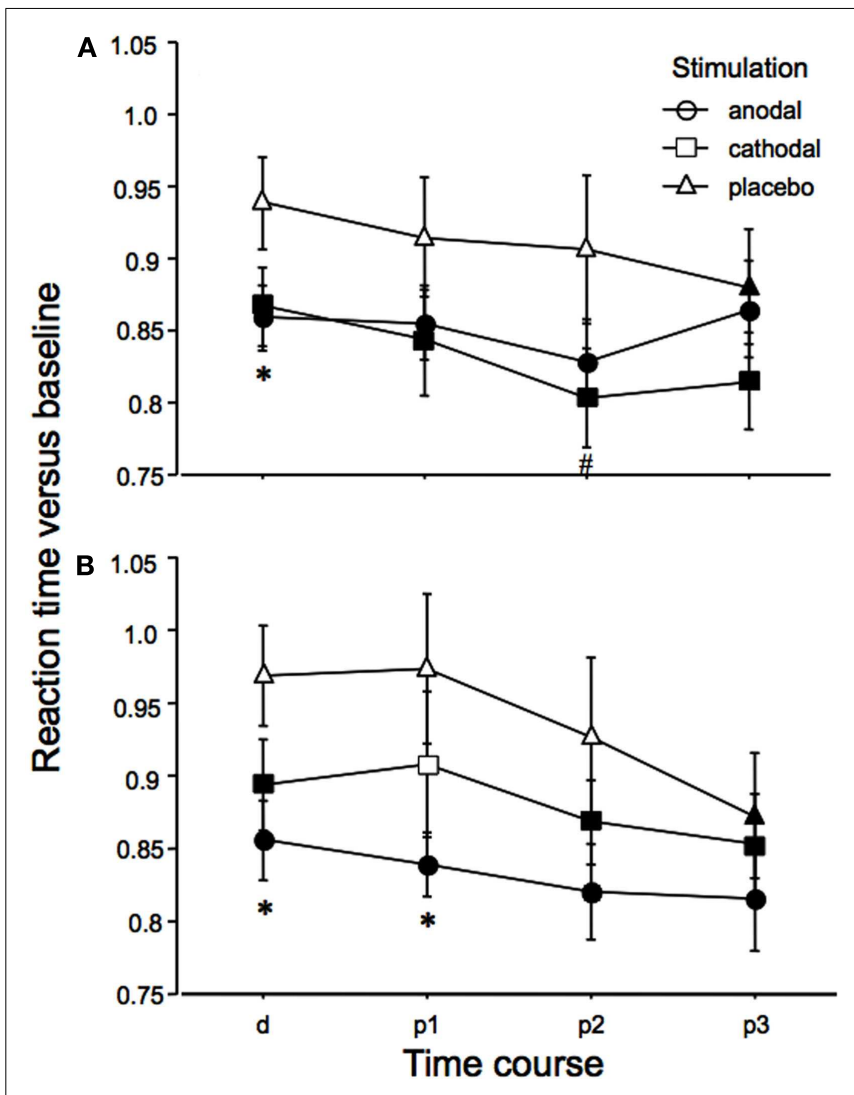

FIGURE 3 | Emotional face identification is modified by tDCS: reaction times. Baseline-standardized reaction times for the identification of the position of negative (A) or positive (B) emotional facial expressions shown on a computer screen are depicted during (d) and after (p1-3; p1 $=$ immediately and 5 min after tDCS, p2 $=10$ and 20 min after tDCS, p3 $=30$ and 60 min after tDCS), anodal, cathodal, and placebo tDCS. Reaction times become faster during the course of the experiment, thus indicating learning of the task in all stimulation and emotional conditions. Under both real stimulation conditions and for both facial expressions, reaction time reductions become earlier significant than under placebo stimulation. Under anodal tDCS, positive emotional facial expressions are faster identified as compared to placebo stimulation during and after tDCS. For emotionally negative facial expressions, anodal tDCS improves perception only during tDCS as compared to placebo stimulation. A minor effect can be seen for cathodal tDCS, as compared to placebo stimulation (p2 only). Filled symbols indicate significant reaction time differences as compared to baseline values, asterisks significant differences between anodal tDCS and placebo tDCS, and hash symbols significant differences between cathodal and placebo tDCS for a given time point (paired, two-tailed $t$-tests, $p<0.05)$. Vertical bars indicate standard error of mean.

\section{Correct answers}

The ANOVA revealed a significant main effect of time (Table 1). As can be seen in Figure 4, this is caused by an increased number of correct answers relative to baseline in the later blocks of the task for all stimulation and facial expression conditions. For the placebo and anodal stimulation condition, but not for cathodal tDCS, this effect is significant during the whole time course of the experiment for the recognition of negative emotional facial expressions. For positive facial expressions, anodal tDCS caused a significant improvement as compared to baseline in the last two measures, 


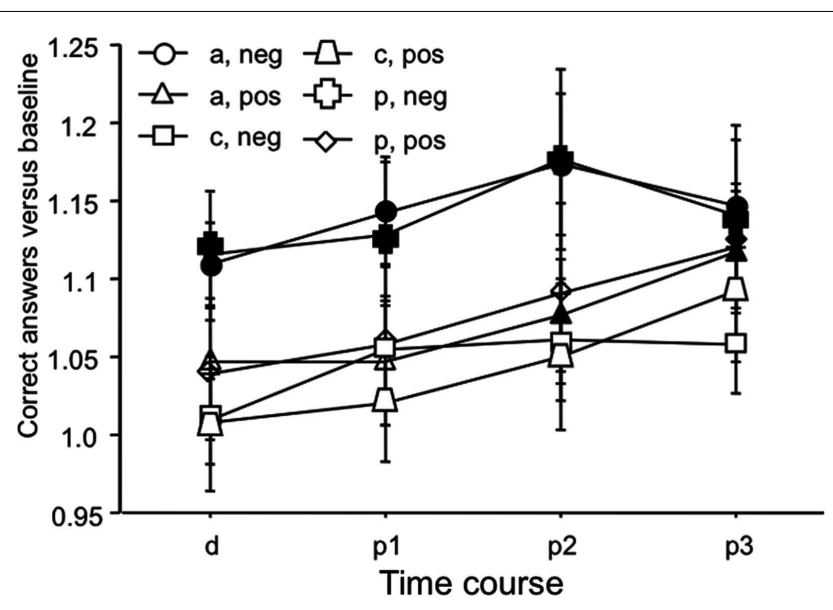

FIGURE 4 | Emotional face identification: number of correct trials. Baseline-standardized mean number of correct trials are depicted during, and after anodal, cathodal, and placebo tDCS (p1-3; p1 = immediately and $5 \mathrm{~min}$ after tDCS, p2 $=10$ and $20 \mathrm{~min}$ after tDCS, p3 $=30$ and $60 \mathrm{~min}$ after tDCS). The number of correct trials increases during the course of the experiment, thus indicating learning of the task in all stimulation and emotional conditions. This effect is significant for negative facial expressions under anodal and placebo tDCS conditions for the whole course of the experiment, but not for cathodal tDCS. For positive facial expressions, anodal tDCS caused a significant improvement as compared to baseline in the last two measures, and placebo tDCS in the last measure. Again under cathodal tDCS facial recognition did not improve significantly. Filled symbols indicate significant reaction time differences as compared to baseline values (paired, two-tailed $t$-tests, $p<0.05$ ). Vertical bars indicate standard error of mean. a, anodal tDCS; $c$, cathodal tDCS; $p$, placebo tDCS; pos, positive emotional facial expression; neg, negative emotional facial expression.

and placebo tDCS in the last measure. Baseline values did not differ significantly between the respective stimulation conditions.

\section{DISCUSSION}

The results of this study suggest that tDCS of the prefrontal cortex has an impact on emotional state-related information processing in healthy subjects. tDCS of the left DLPFC improved emotional face recognition, most markedly for emotionally positive faces, and anodal tDCS. This effect, however, seems to be not accompanied by modifications of subjective emotional state, which was not affected by tDCS in Experiment 1. Therefore, the results are in favor for a dissociation of the impact of tDCS on self-referenced emotional state and state-related information processing in healthy subjects.

\section{MISSING EFFECT OF PREFRONTAL tDCS ON EMOTIONAL STATE IN HEALTHY SUBJECTS}

Anodal and cathodal tDCS of the left DLPFC, combined with antagonistic stimulation of the right supraorbital area, did not modulate emotional state, as rated by an adjective checklist. Positive, negative, and neutral ratings were identical in all conditions. Since negative and neutral - the latter representing lethargy emotional state values were near the minimum throughout the experiment independent from stimulation condition, while positive mood was more in a medium range, one could suspect that social expectancy contributed to these results. However, the SES has been shown to be a reliable and valid instrument to measure emotional state in other studies (Scholz, 2001). This pattern of results is comparable to the effects of left DLPFC rTMS, and another tDCS study (Plazier et al., in press). It might be caused by a kind of ceiling effect preventing a further increase of positive emotional state and a floor effect for negative and lethargic emotional state in healthy subjects with normal activation of the DLPFC.

\section{tDCS MODULATES EMOTION-RELATED INFORMATION PROCESSING}

In general, the effects of tDCS on emotional face identification were relatively low, the results of the respective ANOVAs showed only a trendwise effect of tDCS. We nevertheless conducted exploratory post hoc tests to identify also slight tDCS-related alterations. These, however, should be confirmed in larger studies in future.

During the course of the experiment subjects were able to identify the position of the emotional non-neutral face faster, independent of stimulation condition, or mood quality, i.e., a learning process took place. This performance improvement tended to be larger for the real tDCS conditions. In principle this could be caused by an unspecific arousal effect of real tDCS as compared to placebo stimulation. However it was shown recently that placebo stimulation, as performed in our study, cannot be discerned from real stimulation by the subjects (Gandiga et al., 2006). Furthermore, former studies showed a highly stimulation polarity- and electrode position-dependent effect of tDCS. Moreover, tDCS did not induce arousal in our subjects, as shown by the results of the adjective checklist for the items representing lethargy. Thus unspecific arousal as the result of stimulation seems not a likely explanation. A tDCS-dependent alteration of attention can however not be excluded completely, since tDCS, although of other areas, has been shown to affect attentional processes (Bolognini et al., 2010).

The reaction time results, according to the results of the exploratory post hoc tests, moreover hint to a specific beneficial effect of anodal tDCS on recognition of positive and negative emotional facial expressions as compared to baseline values. This result, which is most probably due to improved information processing by excitability-enhancing anodal tDCS of the DLPFC, is in accordance with former studies. Here an involvement of the left DLPFC in the processing of affective material, especially emotionally valenced faces was described (Herrington et al., 2005; Sergerie et al., 2005; Grimm et al., 2006). For this, the impact of the DLPFC on the evaluation of accumulated information and response selection might be of importance (Badre and Wagner, 2004). Furthermore, as compared to placebo stimulation, the improvement of performance tended to be larger for the emotional positive facial expressions than for the negative ones. This result is in accordance with the fMRI study of Sergerie et al. (2005), where left DLPFC activation was enhanced by emotionally positive and negative faces, but to a larger degree by the positive faces. Thus the results of our study are compatible with the finding that the left DLPFC is involved in the processing of emotional valenced faces in general, but with an additional emphasis on positive emotions. 
For reaction time, also under cathodal tDCS the results of the exploratory post hoc tests show a - somewhat weaker effect for improved performance relative to placebo stimulation. This might be caused by a slight improvement of information processing induced by general network excitability reduction, which has a focusing effect on perception, as demonstrated in former studies (Antal et al., 2004). Alternatively, since due to the electrode arrangement left DLPFC stimulation was inevitably associated with right prefrontal tDCS, and the right prefrontal cortex is also involved in affective information processing (Herrington et al., 2005; Kensinger and Schacter, 2006), the accompanying anodal right prefrontal tDCS might have contributed.

With regard to the number of correct identifications of the emotional facial expression, an increase takes place throughout the course of the experiment, which is significant relative to baseline for the latest measures under anodal and placebo tDCS for positive and negative emotional expressions, as shown by the exploratory post hoc tests. Negative facial expressions were significantly better recognized, as compared to baseline, also in earlier blocks for anodal and placebo stimulation. However, under cathodal tDCS the amount of correctly identified faces did not significantly improve for both emotional qualities relative to baseline. This pattern of results is in favor for a relative decrease of the ability of the subjects to identify emotional facial expressions under a cortical excitability diminution, as delivered by cathodal tDCS. While at first sight this result seems to contradict the reaction time results under cathodal tDCS, it might be explained as follows: the overall excitability reduction will impair the ability to correctly identify a facial expression, but, once identified, enhance reaction time via focusing cortical activity.

\section{GENERAL REMARKS}

Taken together, the results of the experiments are compatible with a dissociation of the effects of left DLPFC stimulation on self-referenced emotional state and emotion-related information processing. Whereas in the healthy subjects emotional state was not modulated, the identification of facial expression of emotions was improved by tDCS. This assumed dissociation is in line with the current state of research, since it has been shown that for the prefrontal cortex, the medial inferior prefrontal cortex is mainly involved in self-referenced emotion, while cognitive functions are localized predominantly in the DLPFC (Steele and Lawrie, 2004). However, since it has also been shown that both areas are functionally overlapping, it makes sense that the DLPFC might be

\section{REFERENCES}

Antal, A., Nitsche, M. A., Kruse, W., Kincses, T. Z., Hoffmann, K. P., and Paulus, W. (2004). Direct current stimulation over V5 enhances visuomotor coordination by improving motion perception in humans. J. Cogn. Neurosci. 16, 521-527.

Badre, D., and Wagner, A. D. (2004). Selection, integration, and conflict monitoring; assessing the nature and generality of prefrontal cognitive

involved in cognitive processing of emotional material. A limitation of Experiment 2 is that emotional state has not been tested in this experiment. Thus it cannot be ruled out completely that the emotional state of the subjects differed systematically between the respective experimental sessions, and that such differences have affected the results of this experiment.

With regard to the facial recognition task, the results of our study are furthermore in favor for a larger involvement of the left DLPFC in the identification of affective positive than of affective negative faces. This is in line with the notion that the left hemisphere is more involved in positive than in negative emotions. It is proposed that left hemispheric brain tumors and epilepsies are associated with depression, while tumors of the right hemisphere cause euphoric mood (Perini, 1986; Belyi, 1987). Moreover, happy mood and presentation of emotionally positive stimuli produce stronger activation of the left prefrontal cortex in healthy subjects (Habel et al., 2005; Herrington et al., 2005; Sergerie et al., 2005). Facial recognition, however, was also improved - but to a smaller degree - for emotionally negative faces by tDCS. This effect might be caused by an additional involvement of the DLPFC in facial recognition, which is independent from a specific emotional quality (Kilts et al., 2003; Sergerie et al., 2005; Pavuluri et al., 2006; Schutter and van Honk, 2006).

The failure of excitability-enhancing tDCS of the left DLPFC to induce positive mood in healthy subjects is surprising at first sight, because this stimulation protocol improved mood in a group of depressed subjects relevantly in a former study (Fregni et al., 2006). It is however in accordance with the results of a recently published study of another group (Plazier et al., in press). Moreover, also rTMS protocols that were effective to improve mood in depression failed to have the same effect in healthy subjects (Mosimann et al., 2000; Baeken et al., 2006).

The present study delivers indirect hints for a dissociation of emotion-related information processing and self-referenced emotional state. The results are in favor for the hypothesis that the DLPFC might be more involved in the cognitive aspects of emotional processing. To influence emotional state more directly by external stimulation techniques, it might be promising to study other prefrontal areas, such as the inferior-medial prefrontal cortex, which seem to be critically involved in the production of emotions (Steele and Lawrie, 2004) in future studies.

\section{ACKNOWLEDGMENTS}

This study was partly funded by the BMBF, Bernstein-Center for Computational Neuroscience, Goettingen.

Belyi, B. I. (1987). Mental impairment in unilateral frontal tumours: role of the laterality of the lesion. Int. J. Neurosci. 32, 799-810.

Boggio, P. S., Bermpohl, F., Vergara, A. O., Muniz, A. L., Nahas, F. H., Leme, P. B., Rigonatti, S. P., and Fregni, F. (2007). Go-no-go task performance improvement after anodal transcranial DC stimulation of the left dorsolateral prefrontal cortex in major depression. J. Affect. Disord. 101, 91-98.
Boggio, P. S., Zaghi, S., and Fregni, F. (2009). Modulation of emotions associated with images of human pain using transcranial direct current stimulation (tDCS). Neuropsychologia 47, 212-217.

Bolognini, N., Fregni, F., Casati, C., Olgiati, E., and Vallar, G. (2010). Brain polarization of parietal cortex augments training-induced improvement of visual exploratory and attentional skills. Brain Res. 1349, 76-89. 
Ekman, P. (1999). "Basic emotions," in Handbook of Cognition and Emotion, eds T. Dalgleish and M. J. Power (New York: John Wiley and Sons), 45-60.

Ekman, P., and Friesen, W. V. (1975). Unmasking the Face. Englewood Cliffs, NJ: Prentice-Hall.

Ekman, P., and Friesen, W. V. (1976). Pictures of Facial Affect. Palo Alto: Consulting Psychologist Press.

Ellis, H. C., and Moore, B. A. (1999). "Mood and memory," in Handbook of Cognition, and Emotion, eds T. Dalgleish and M. J. Power (New York: John Wiley and Sons), 191-210.

Fregni, F., Boggio, P. S., Nitsche, M. A., Marcolin, M. A., Rigonatti, S. P., and Pascual-Leone, A. (2006). Treatment of major depression with transcranial direct current stimulation. Bipolar Disord. 8, 203-204.

Gandiga, P. C., Hummel, F. C., and Cohen, L. G. (2006). Transcranial DC stimulation (tDCS): a tool for double-blind sham-controlled clinical studies in brain stimulation. Clin. Neurophysiol. 117, 845-850.

Grimm, S., Schmidt, C. F., Bermpohl, F., Heinzel, A., Dahlem, Y., Wyss, M., Hell, D., Boesiger, P., Boeker, H., and Northoff, G. (2006). Segregated neural representation of distinct emotion dimensions in the prefrontal cortex-an fMRI study. Neuroimage 30, 325-340.

Habel, U., Klein, M., Kellermann, T., Shah, N. J., and Schneider, F. (2005). Same or different? Neural correlates of happy and sad mood in healthy males. Neuroimage 26, 206-214.

Hamilton, M. (1960). A rating scale for depression. J. Neurol. Neurosurg. Psychiatr. 23, 56-62.

Hampel, R. (1977). Adjektiv-Skalen zur Einschätzung der Stimmung (SES). Diagnostica 23, 43-60.

Herrington, J. D., Mohanty, A., Koven, N. S., Fisher, J. E., Stewart, J. L., Banich, M. T., Webb, A. G., Miller, G. A., and Heller, W. (2005).
Emotion-modulated performance and activity in left dorsolateral prefrontal cortex. Emotion 5, 200-207.

Kensinger, E. A., and Schacter, D. L. (2006). Processing emotional pictures and words: effects of valence and arousal. Cogn. Affect. Behav. Neurosci. 6, 110-126.

Kilts, C. D., Egan, G., Gideon, D. A., Ely, T. D., and Hoffman, J. M. (2003). Dissociable neural pathways are involved in the recognition of emotion in static and dynamic facial expressions. Neuroimage 18, 156-168.

Maeoka, H., Matsuo, A., Hiyamizu, M., Morioka, S., and Ando, H. (2012). Influence of transcranial direct current stimulation of the (dorsolateral) prefrontal cortex on pain related emotions: a study using electroencephalographic power spectrum analysis. Neurosci. Lett. 512, 12-16.

Mitchell, P. B., and Loo, C. K. (2006). Transcranial magnetic stimulation for depression. Aust. N. Z. J. Psychiatry 40, 406-413.

Mosimann, U. P., Rihs, T. A., Engeler, J., Fisch, H., and Schlaepfer, T. E. (2000). Mood effects of repetitive transcranial magnetic stimulation of left prefrontal cortex in healthy volunteers. Psychiatry Res. 94, 251-256.

Nitsche, M. A., Nitsche, M. S., Klein, C. C., Tergau, F., Rothwell, J. C., and Paulus, W. (2003a). Level of action of cathodal DC polarisation induced inhibition of the human motor cortex. Clin. Neurophysiol. 114, 600-604.

Nitsche, M. A., Fricke, K., Henschke, U., Schlitterlau, A., Liebetanz, D., Lang, N., Henning, S., Tergau, F., and Paulus, W. (2003b). Pharmacological modulation of cortical excitability shifts induced by transcranial direct current stimulation in humans. J. Physiol. 553, 293-301.

Nitsche, M. A., and Paulus, W. (2000). Excitability changes induced in the human motor cortex by weak transcranial direct current stimulation. J. Physiol. 527, 633-639.

Nitsche, M. A., and Paulus, W. (2001). Sustained excitability elevations induced by transcranial DC motor cortex stimulation in humans. Neurology 57, 1899-1901.

Pavuluri, M. N., O'Connor, M. M., Harral, E., and Sweeney, J. A. (2006). Affective neural circuitry during facial emotion processing in pediatric bipolar disorder. Biol. Psychiatry 62, 158-167.

Perini, G. I. (1986). Emotions and personality in complex partial seizures. Psychother. Psychosom. 45, 141-148.

Phan, K. L., Wager, T., Taylor, S. F. and Liberzon, I. (2002). Functional neuroanatomy of emotion: a metaanalysis of emotion activation studies in PET and fMRI. Neuroimage 16, 331-348.

Plazier, M., Joos, K., Vanneste, S. Ost, J., and De Ridder, D. (in press). Bifrontal and bioccipital transcranial direct current stimulation (tDCS) does not induce mood changes in healthy volunteers: a placebo controlled study. Brain Stimul.

Robinson, R. G., and Lipsey, J. R. (1985). Cerebral localization of emotion based on clinical-neuropathological correlations: methodological issues. Psychiatr. Dev. 3, 335-347.

Scholz, O. B. (2001). Kurz- und mittelfristige effekte hypnotischer stimmungsinduktion. Z. Psychol. 209, 118-136.

Schutter, D. J., and van Honk, J. (2005). A framework for targeting alternative brain regions with repetitive transcranial magnetic stimulation in the treatment of depression. J. Psychiatry Neurosci. 30, 91-97.

Schutter, D. J., and van Honk, J. (2006). Increased positive emotional memory after repetitive transcranial magnetic stimulation over the orbitofrontal cortex. J. Psychiatry Neurosci. 31, 101-104.
Sergerie, K., Lepage, M., and Armony, J. L. (2005). A face to remember: emotional expression modulates prefrontal activity during memory formation. Neuroimage 24, 580-585.

Steele, J. D., and Lawrie, S. M. (2004). Segregation of cognitive and emotional function in the prefrontal cortex: a stereotactic meta-analysis. Neuroimage 21, 868-875.

Ueda, K., Okamoto, Y., Okada, G., Yamashita, H., Hori, T., and Yamawaki, S. (2003). Brain activity during expectancy of emotional stimuli: an fMRI study. Neuroreport 14, 51-55.

Watson, D., and Clark, L. A. (1988). Development and validation of brief measures of positive and negative affect: the panas scales. J. Pers. Soc. Psychol. 54, 1063-1070.

Conflict of Interest Statement: The authors declare that the research was conducted in the absence of any commercial or financial relationships that could be construed as a potential conflict of interest.

Received: 24 February 2012; accepted: 25 May 2012; published online: 18 June 2012.

Citation: Nitsche MA, Koschack J, Pohlers H, Hullemann S, Paulus W and Happe S (2012) Effects of frontal transcranial direct current stimulation on emotional state and processing in healthy humans. Front. Psychiatry 3:58. doi: 10.3389/fpsyt.2012.00058

This article was submitted to Frontiers in Neuropsychiatric Imaging and Stimulation, a specialty of Frontiers in Psychiatry. Copyright (c) 2012 Nitsche, Koschack, Pohlers, Hullemann, Paulus and Happe. This is an open-access article distributed under the terms of the Creative Commons Attribution Non Commercial License, which permits non-commercial use, distribution, and reproduction in other forums, provided the original authors and source are credited. 


\section{APPENDIX}

Table A1 | Here the results of the post hoc Student's $\boldsymbol{t}$-tests conducted on standardized reaction times and error rate of Experiment 2 are shown.

\begin{tabular}{|c|c|c|c|c|c|}
\hline & Mean value & Confidence intervals - lower bound & Confidence intervals - upper bound & $t$-Value & $P$ \\
\hline \multicolumn{6}{|c|}{ STANDARDIZED REACTION TIMES } \\
\hline \multicolumn{6}{|l|}{ vs $B L$} \\
\hline p1 a pos & 0.16 & 0.11 & 0.2 & 7.21 & $<0.001$ \\
\hline p2 a pos & 0.18 & 0.11 & 0.25 & 5.42 & $<0.001$ \\
\hline p1 a neg & 0.15 & 0.10 & 0.20 & 6.02 & $<0.001$ \\
\hline p2 a neg & 0.17 & 0.11 & 0.23 & 5.91 & $<0.001$ \\
\hline p3 a neg & 0.14 & 0.07 & 0.21 & 4.03 & 0.001 \\
\hline dc pos & 0.11 & 0.04 & 0.17 & 3.39 & 0.03 \\
\hline p1 c pos & 0.09 & 0.01 & 0.19 & 1.84 & 0.081 \\
\hline p2 c neg & 0.20 & 0.12 & 0.27 & 5.64 & 0.001 \\
\hline p3 c neg & 0.19 & 0.11 & 0.26 & 5.48 & $<0.001$ \\
\hline ds pos & 0.03 & 0.04 & 0.10 & 0.91 & 0.37 \\
\hline p1 s pos & 0.03 & 0.08 & 0.13 & 0.52 & 0.61 \\
\hline p2 s pos & 0.07 & 0.04 & 0.19 & 1.35 & 0.19 \\
\hline p3 s pos & 0.13 & 0.04 & 0.22 & 2.95 & 0.008 \\
\hline ds neg & 0.06 & 0.01 & 0.13 & 1.91 & 0.071 \\
\hline p1 s neg & 0.09 & 0.01 & 0.17 & 2.07 & 0.051 \\
\hline p2 s neg & 0.09 & 0.01 & 0.20 & 1.81 & 0.085 \\
\hline p3 s neg & 0.12 & 0.04 & 0.20 & 3.04 & 0.007 \\
\hline p3 a neg & 0.02 & 0.10 & 0.07 & 0.38 & 0.708 \\
\hline$d c$ pos & 0.08 & 0.18 & 0.03 & 1.50 & 0.150 \\
\hline p1 c pos & 0.06 & 0.20 & 0.07 & 1.00 & 0.329 \\
\hline p2 c pos & 0.06 & 0.17 & 0.06 & 1.07 & 0.299 \\
\hline p3 c pos & 0.01 & 0.10 & 0.06 & 0.49 & 0.628 \\
\hline$d c$ neg & 0.07 & 0.17 & 0.02 & 1.57 & 0.132 \\
\hline p1 c neg & 0.07 & 0.17 & 0.03 & 1.47 & 0.157 \\
\hline p2 c neg & 0.1 & 0.20 & 0.01 & 2.14 & 0.045 \\
\hline p3 c neg & 0.07 & 0.14 & 0.01 & 1.71 & 0.104 \\
\hline \multicolumn{6}{|c|}{ STANDARDIZED ERRORS } \\
\hline \multicolumn{6}{|l|}{ vs $B L$} \\
\hline$d$ a pos & 0.05 & 0.13 & 0.04 & 1.18 & 0.253 \\
\hline p1 a pos & 0.05 & 0.13 & 0.04 & 1.16 & 0.260 \\
\hline p2 a pos & 0.08 & 0.15 & 0.01 & 2.13 & 0.045 \\
\hline p3 a pos & 0.12 & 0.20 & 0.03 & 3.00 & 0.008 \\
\hline
\end{tabular}


Table A1 | Continued

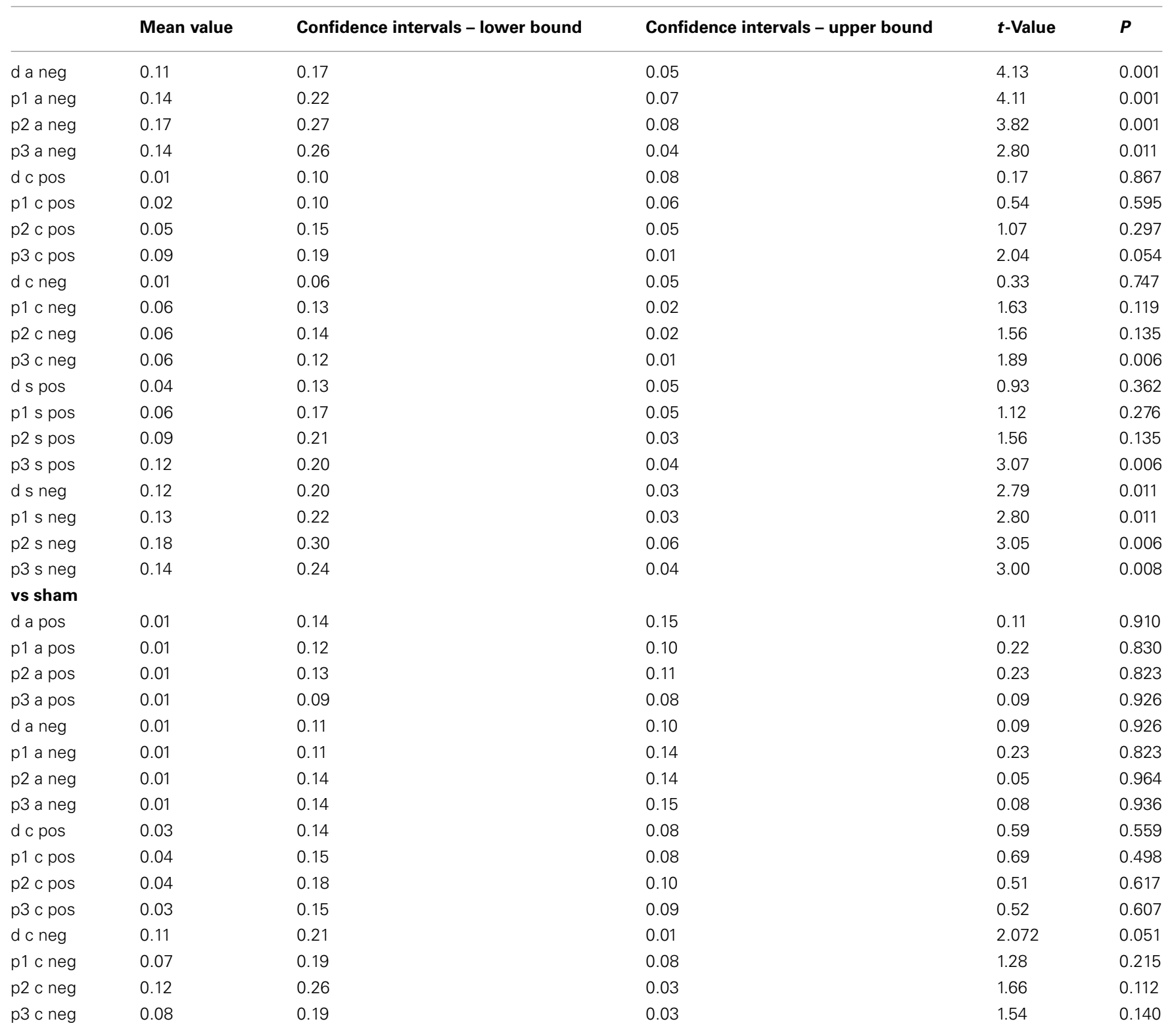

$d$, During $t D C S ; p 1$, first measure post-tDCS; 2 , second measure post-tDCS; $p 3$, third measure post-tDCS; a, anodal tDCS; $c$, cathodal tDCS; s, sham tDCS. 